\title{
High-resolution integrated modelling of the spatial dynamics of urban and regional systems
}

\author{
R. White ${ }^{\mathrm{a}, *}$, G. Engelen ${ }^{\mathrm{b}}$ \\ ${ }^{a}$ Department of Geography, Memorial University of Newfoundland, St. John's, Newfoundland, \\ Canada $A 1 B 3 X 9$ \\ ${ }^{\mathrm{b}}$ Research Institute for Knowledge Systems, Postbus 463, 6200 AL Maastricht, The Netherlands
}

Received 5 May 1999; received in revised form 17 December 1999; accepted 21 January 2000

\begin{abstract}
An emerging branch of geocomputing involves the modelling of spatial processes. A variety of techniques are being used, the most important being traditional regionalized system dynamics approaches, multi-agent systems, and cellular automata (CA). The techniques are frequently combined to model processes operating at different spatial scales. Urban and regional models based on CA give good representations of the spatial dynamics of land use. In a current application, a cellular model of The Netherlands at $500 \mathrm{~m}$ resolution is driven by a macro-scale dynamical spatial interaction model defined on 40 economic regions; this model is in turn driven by national planning projections and policy goals. Given the national totals, the macro-scale model generates regional demands for population and a number of economic activities. These demands are translated into demands for cell space, which the CA then attempts to locate. In turn, information on conditions at the cellular level, such as the quantity and quality of land available to various activities and actual densities at the cellular scale, are returned to the regional model to modify parameter values there. Linking the two models operating at the two scales improves the performance of both. The results of high-resolution modelling of spatial dynamics raise several methodological issues. One of the most pressing concerns evaluation of the results. Another issue concerns predictability. To the extent that these models capture the evolving nature of real cities and regions, they cannot be strictly predictive. (C) 2000 Elsevier Science Ltd. All rights reserved.
\end{abstract}

Keywords: Cellular automata; Land use; Integrated models; Process models; Spatial dynamics

\footnotetext{
* Corresponding author. Fax: +1-709-737-3119.
}

E-mail address: roger@plato.ucs.mun.ca (R. White). 


\section{Introduction}

Most geocomputation currently deals with the processing of spatial data in order to show us the world as it is; but of course the world can be seen from many points of view, and one of the great strengths of geographical information systems (GIS) is that it allows us continually to reconfigure the data in the ways that are most appropriate for our changing needs and points of view. Spatial statistics by and large tend to perform the same sort of task, but in a more abstract way, allowing us to make generalizations about what we see in the data, to extract hypotheses from it, or, finally, to use it to test hypotheses.

But the data that is stored and processed in a GIS contains, so to speak, the seeds of its own destruction. The patterns of land cover and land use, and of social, economic, and demographic characteristics, constantly change, both because the spatial structures are themselves inherently unstable, and because they are typically exposed to external phenomena that also force change. This problem is dealt with by programmes for periodic data collection and updating, but such a response is not sufficient for all purposes, since at best it gives us only a regularly updated picture of current conditions. Planners and decision makers need to know not only the current state of affairs, they also require some idea of future conditions. Ideally they would like to be able to see the possible consequences of the plans and policies they may have under consideration. These considerations point to another class of geocomputation techniques - specifically, predictive computational models. To the extent that these models embody dynamics capturing the endogenous instabilities of existing spatial configurations, they can be thought of as dynamical GIS.

The favoured techniques for implementing high-resolution models of spatial dynamics are cellular automata (CA) and multi-agent systems (MA). CA are attractive for a number of reasons:

1. they are inherently spatial; typically they are defined on a raster cell space and are thus compatible, or can be made compatible, with most spatial data sets;

2. they are dynamic, and can thus represent spatial processes in a direct way;

3. they are highly adaptable - they can be set up to represent a very wide range of situations and processes;

4. they are rule based, and can thus capture a wide variety of spatial behaviours;

5. they are simple, and thus computationally efficient; and

6. in spite of their simplicity, they can exhibit extraordinarily rich behaviour; some simple CA have been shown to be formally equivalent to a Turing machine, i.e. these CA can represent and execute any possible algorithm.

MA systems also have attractive features:

1. they provide a straightforward way to represent spatial entities or actors having relatively complex properties or behaviours;

2. they provide inheritance of properties from class to subclass, so that they represent hierarchical systems in a natural way; and 
3. they capture directly the interactive properties of many natural and human systems, as well as the complex system behaviour that emerges from this interaction.

The two approaches overlap to some degree; indeed, CA are occasionally considered to be a type of MA system. In the context of geocomputation, MAs are most commonly used together with CA to represent, for example, individuals moving around in a cell space endowed with its own CA dynamics (Benenson, 1998; Portugali \& Benenson, 1997), or to represent clusters of cells which may be generated by the dynamics of a CA, and which as a cluster maybe acquire emergent properties. However, MAs can be used on their own, e.g. to model the dynamics of an urban system containing a variety of types of urban centres (Bura, Guerin-Pace, Mathian, Pumain \& Sanders, 1996; Sanders, Pumain \& Mathian, 1997).

In this paper we focus on CA. Tobler (1979) was the first to suggest the use of CA in geographical modelling. He was followed by Phipps $(1989,1992)$, who focused on theoretical problems of cluster formation, and Couclelis (1985, 1988, 1989), who in this early work used the technique to explore theoretical issues such as complexity and structure formation. In more recent work, both (Couclelis, 1997; Phipps \& Langlois, 1997) have continued to pursue theoretical problems associated with CA representations of geographical systems. Cecchini and Viola (1990) were also among the first to adopt CA for spatial modelling, and have continued to work in this area (Cecchini, 1996). Papini and Rabino (1997) have used CA to model urban form. Since CA can be considered an extension of GIS, in which a dynamics is imposed on the data structures, the link between the two has attracted a certain amount of attention: Itami (1994), Wagner (1997), White and Engelen (1994), and Wu (1998b) have all considered this issue. Theobald and Gross (1994) have gone further and discussed the integration of GIS, CA, and System Dynamics techniques (Stella); they thus support the approach taken in this paper, which emphasizes integrated modelling using all three types of approaches.

More recently, there have been a number of applications of CA that are aimed at developing the technique as one which can be applied to practical problems in such areas as land use planning, social policy, and impact assessment. Clarke, Hoppen and Gaydos (1997) used a CA to model the historical development of urbanization in the San Francisco Bay area. Batty and Xie (1994, 1996) and Xie (1996) developed several urban models, one of which was applied to the development of a residential area on the fringe of Buffalo, USA. The work of Wu (1998b) is also directed at developing planning applications. Portugali and Benenson $(1995,1997)$ and Portugali, Benenson and Omer $(1994,1997)$ have emphasized empirical realism in highly detailed models which combine land use dynamics with models of socio-economic and ethnic group formation; these are applied in Tel Aviv, Israel. Finally, Engelen, White and Uljee (1997), Englelen, Uljee and White (1997), Engelen, White, Uljee and Wargnies (1996), Uljee, Engelen and White (1996), White and Engelen (1993, 1997a, b), and White, Engelen and Uljee (1997, 1999) have developed several CA and CA-based integrated models designed as prototypes of Spatial DecisionSupport Systems for urban and regional planning and impact analysis (fully functional 
demos of several of these models can be downloaded from www.riks.nl/RiksGeo/ freestuff.htm).

The cumulative effect of this work is to demonstrate that CA are remarkably effective at generating realistic simulations of both land use patterns and other spatial structures. Unlike conventional System Dynamics techniques, they have proven able to handle high-resolution applications easily, and thus to combine the precision of high-quality data sets typically resident in GIS with the realism of dynamics to yield convincing predictions of the future states of spatial systems.

\section{Characteristics of $\mathbf{C A}$}

CA are perhaps the simplest type of dynamic spatial model. Essentially, they consist of:

1. a grid or raster space;

2. a set of states which characterize the grid cells;

3. a definition of the neighbourhood of a cell;

4. a set of transition rules that determine the state transitions of each cell as a function of the states of neighbouring cells; and

5. a sequence of discrete time steps, with all cells updated simultaneously.

The most basic CA, like Game of Life and the one-dimensional CA used for fundamental research in the properties of dynamical systems, embody these characteristics in a straightforward way. In Game of Life, for example, the cell space is a twodimensional rectilinear grid, there are two possible cell states, alive and dead, and the transition rules are simple, e.g. if a dead cell has exactly three live cells in its eight-cell neighbourhood, then at the next iteration it changes state to alive. But the CA that have been developed to model geographical systems typically are much more complex, and relax these defining characteristics in a number of ways in order to come as close as possible to a faithful representation of the system being modelled. Thus, it is worthwhile to examine some of these variations on the CA theme and the reasons why they are useful in a geocomputational setting.

\subsection{The grid space}

Grid space is typically assumed to be two dimensional, rectilinear, and homogeneous; but these assumptions are frequently dropped. While it is clear that for most geographical applications involving land use and land cover it is natural to use a two-dimensional grid, for some applications it may be more straightforward to use grids with other dimensions. Some of the most advanced urban traffic models, for example (Nagel, Rasmussen \& Barrett, 1997), have been developed using onedimensional CA, although in these models the linear CA are concatenated in order to represent the network structure of the road system. Similarly, it might be useful to 
model dense, multi-story urban areas with three-dimensional CA, although to date this has not been done.

Rectilinear grid systems have obvious advantages both in terms of compatibility with raster-based data systems and in terms of computational efficiency. However, the regular structure in principle may introduce artifacts into the spatial structures generated by the CA, and so some authors have proposed using a hexagonal tiling or even randomizing the grid coordinates in order to minimize or eliminate the macroscopic asymmetries that characterize a square lattice. So far there seem to be no geographical applications that make use of such a randomization procedure.

There are other reasons to depart from a regular grid. In current applications, cell sizes range from $500 \mathrm{~m}$ down to tens of metres. If the space to be modelled is already subdivided into functionally relevant units that approximate the scale of the grid, then these units will provide a better representation of the space than will grid cells. For example, if land use modelling is to be carried out at a resolution approximating the scale of cadastral or land ownership units, then using cadastral units rather than grid cells will result in a better conformation of model results to actual land use boundaries, although there will of course be a loss of computational efficiency, as well as a complication of the definition of the 'cell' neighbourhood. Batty and Xie (1994) have employed cadastral units in a model of land use changes in a suburban area of Buffalo, USA.

For typical geographical applications, the most important grid space assumption to relax is that of homogeneity. Realistically, the space on which the state dynamics is played out is far from homogeneous. If the states represent land use, then it is clear that a number of factors other than the land use of neighbouring cells - factors such as slope, soil quality, and zoning regulations - may be important determinants of land use change. Such factors can be thought of as characterizing the cell space itself. In this view, then, each cell is characterized by an intrinsic suitability for each particular land use, or state, which acts in addition to the standard cellular neighbourhood effect in determining the cell state transitions. These suitabilities may also include non-local factors that are not represented in the cell space neighbourhood, such as accessibility to a transportation network or to key points in the region like a city centre or an airport.

A number of CA models have been developed in which the cell space is inhomogeneous (Engelen, White \& Uljee, 1993; White \& Engelen, 1997a; White, Engelen \& Uljee, 1997). The idea of including suitabilities in the characterization of the cell space emphasizes the importance of the link between CA modelling and GIS, since the suitabilities are typically calculated within a GIS and imported into the cellular model. However, when suitabilities consist of weighted sums of cell characteristics, it is frequently the case that the proper values of the weights are not known. In this case determination of the precise values of the weights becomes part of the calibration process of the $\mathrm{CA}$, and it is convenient to have a software tool that sits between the GIS and the CA and facilitates the process of calibrating the weights. In any case, the weights, or more accurately, the suitabilities determined by them, form part of the boundary conditions of the CA, i.e. they are read in during the initialization of the model and remain fixed during execution. In principle the weights might also 
be altered dynamically within a CA-based model, although apparently no such model has yet been implemented.

\subsection{The cell states}

Cell states most commonly represent land cover and land use, but may be used to represent any spatially distributed variable for the purpose of modelling its spatial dynamics. For example, cell states may be used to represent population density levels $(\mathrm{Wu}, 1998 \mathrm{a})$. In this approach the transition rules add population to cells so that each cell is characterized by a population count that changes with each iteration. Since cell sizes are uniform, the cell values in fact represent population density. Cell states may also consist of vectors representing a number of features. Portugali and Benenson (1995), for example, use cell state vectors to represent ethnic and socio-economic status, demographic attributes, and attitudes towards other groups in their model of neighbourhood and attitude formation. In CA models of natural systems, cell states are used to represent such factors as sediment load in seawater, groundwater levels, and soil moisture. We mention these to emphasize the compatibility between CA models of human and natural systems.

\subsection{The neighbourhood}

In keeping with the spirit of simplicity of CA, applications most often adopt either the Von Neumann neighbourhood (consisting of the four cells adjacent to the sides of the cell) or the Moore neighbourhood (the eight adjacent cells). For most physical systems, these are clearly the most appropriate definitions, since such systems typically have only local causation (e.g. groundwater must first flow through adjacent cells before it can reach more distant ones). However, in the case of human systems, the idea of locality may be much larger, since people and institutions are aware of their surroundings in a wider space. Thus, it is desirable to define a neighbourhood large enough to capture the operational range of the local processes being modelled by the CA. In the case of land use changes, local or neighbourhood effects may extend to a distance of a kilometre or more. Hence, in some models (e.g. Uljee, Engelen \& White, 1996) the neighbourhood is defined as all cells within a radius of eight cells, an area containing 196 cells. Of course, if the logic of the problem requires, it would be possible to define non-local neighbourhoods, e.g. the neighbourhood might be defined as consisting of cells with which the given cell was linked in some functional sense, without regard to the distance of the cells. Kauffman (1989) has used this kind of neighbourhood in his work with random Boolean networks, which may be regarded as CA with non-local, randomly defined neighbourhoods.

\subsection{The transition rules}

The transition rules are the heart of a CA. They represent the logic of the process which is being modelled, and thus determine the spatial dynamics which result. Since they are as various as the processes they represent, it is difficult to generalize about 
them. They may be simple, as in Game of Life or spatial voting models (a cell takes the state of the majority state in its neighbourhood), or complex; in the limit the 'rule' may consist of an entire sub-model. Rules developed to apply to neighbourhoods with a large cell radius will typically represent local spatial processes that include a distance-decay effect. For example, a rule relating the future land use of a cell to the actual land uses within an eight-cell radius will represent the attraction and repulsion effects of the various land uses in the neighbourhood, but with an attenuation of the effect of the more distant cells. In models of human systems it is usually appropriate, or even necessary, to introduce a stochastic element into the transition process in order to capture the effects of imperfect information and differences among individuals.

\subsection{The time step}

In CA models time is normally discrete, with a simultaneous updating of all cell states after the rules have been applied to each cell using the current configuration. For many applications the appropriate time step is a matter of convenience; e.g. iterations representing 1 year may be adequate in a model of land use change, whereas a model of traffic flow would require time steps of seconds or minutes. One of the most promising roles for CA is in integrated modelling, where several models are linked dynamically and run as one; in these cases the time steps must be defined in such a way as to be compatible. For practical purposes, this requires nested time scales, with each discrete time scale being an integral multiple of the next slower scale.

It is also possible to substitute sequential for simultaneous updating. This is desirable when the process being modelled has relatively few discrete events per time step, as, for example, in the case of a high-resolution model of an urban land market, where individual parcels are put on the market, bid for, and sold (Dong, 2000). In the case of constrained CA, discussed below, sequential updating may also have the advantage of simplifying the problem of attaining appropriate numbers of cells in the various states. This is the primary motivation for using a sequentially updated version of a Boltzman probability transition rule in an urban land use model being developed at Los Alamos National Laboratories. However, sequential updating is clearly only feasible when it is possible to find an appropriate means for selecting the cells to be updated, one that is in accord with the nature of the process being modelled.

\section{Constrained $\mathrm{CA}$ and integrated modelling}

In a pure CA the number of cells in a particular state is determined endogenously by the cellular dynamics. However, this is not realistic for many geographical applications, since usually the number of cells is determined by the level of demand for the activity which is carried out on the cells. Consequently, for most applications, in order to achieve a realistic representation of the system, the cellular model 
is constrained to generate particular numbers of cells in each state, with the target cell numbers determined exogenously, often by another model.

Introducing constraints requires two problems to be solved:

1. numerical values for the constraints must be acquired or generated; and

2. the constraints must be incorporated into the transition rules in such a way as to ensure that they are satisfied without violating the logic of the local processes modelled by the CA.

The second problem is in many ways the more difficult, but it is technical in nature, and its solution depends largely on the details of the particular model; we will not discuss it further here. The first problem, however, is really an opportunity for integrated modelling. For many applications, constraints may simply be supplied as a file to be read during execution time, e.g. the required number of cells of each land use type or the total urban population may be read in at each iteration. However, the constraints may also be generated during run time by one or more linked models. In this case the linked models are usually of interest in their own right, and if the links between them and the CA are two-way, then the constraining models are in turn constrained or modified by input from the cellular-level model.

The constraint models may themselves be regionalized, so that they have their own macro-scale spatial dynamics and provide regionalized constraints to the CA. In an integrated land-use model of The Netherlands, for example, a regionalized economic-demographic model provides demands for cell space for each activity in each of 40 regions, at each iteration. The CA then attempts to achieve these desired totals for the various cell states in each region. But the more desirable a region is, the more difficult it is to satisfy the cell demands. In this case, densities in the region increase, and as higher densities are associated with higher land prices, the region becomes relatively less attractive; specifically, the higher densities are returned to the demographic-economic model where they lower the attractivity of the region in the macro-scale dynamics. As a result of these links, the macro-scale model effectively incorporates high-resolution suitability and land use data resident at the cellular level. This model is discussed in greater detail in the next section.

In general, the possibilities for fully integrated modelling are particularly promising in the area of human-natural systems, where to date there has been very little progress in integrating socio-economic models of human systems with models of natural systems. Most economic and demographic models are either completely aspatial, or are regionalized to relatively large spatial units like provinces. Models of natural systems, on the other hand, e.g. groundwater, soil loss, or ecosystem models, typically are fully spatial, and often of relatively high resolution. There is thus a basic spatial incompatibility between the two types of model. On the ground, the interactions between the two types of systems tend to be place-specific: the population of a province may grow by a certain amount, but the additional people will live in housing built on specific sites and thus modify or destroy particular habitat. Inserting a constrained CA-based model as a link between the two types of model provides the necessary compatibility of spatial scales. This integrating function is potentially one of the most valuable aspects of cellular modelling. 
Several CA-based integrated models are currently under development. One of these, the Urban Security project at Los Alamos National Laboratories (USA) is integrating several existing models, including a high-resolution earthquake destructiveness simulator, using a CA-based urban land use model in order to simulate both the short run impacts of earthquake events and subsequent urban regrowth.

Another, the MODULUS project, sponsored by the Science, Research and Development Directorate (DG-XII) of the European Commission, has as its objective the integration of a number of models that were developed in previous ECfunded research projects. The individual models include a rainfall and solar radiation simulator, a hydrology and soil moisture model, natural vegetation and crop growth models, aquifer and groundwater pumping models, socio-economic scenarios, and an agricultural decision-making model. Some of these, like the rainfall, solar radiation, hydrology and crop growth cluster, were developed as linked models, but others were developed by groups working at several different institutions and were not designed for compatibility. Now they are being linked, so that, for example, the hydrology and aquifer models provide input into the groundwater pumping model, which in turn is linked via an irrigation model back to the hydrology. However, the links between the socio-economic and decision-making modules on the one hand, and the environmental and vegetation models on the other are through a CA-based land use/land cover model. The result is expected to be a spatial decision-support system that will be used by local decision makers in the two Mediterranean regions being modelled.

Finally, the same generic CA land use model used in MODULUS is the basis of an integrated model developed by the Research Institute for Knowledge Systems for RIVM, the environmental research institution of the Dutch government (Engelen, White \& Uljee, 1998; RIVM, 1998). Because this is likely to be the first CA-based integrated model actually to be used by planners and policy makers, we describe it in more detail in the following section.

\section{A CA-based integrated model for land use policy development}

The LeefOmgevingsVerkenner (Environment Explorer) is an integrated model of both land use and the regional distribution of population and economic activity in The Netherlands. It integrates a CA land use model with a regionalized, spatialinteraction based economic-demographic location model. The model covers the whole of The Netherlands; the CA has a resolution of $500 \mathrm{~m}$, while the macro-scale model operates on 40 urban-centred economic regions, or COROPS. The model is driven by national projections for population and sectoral economic activity; there are three sets of projections corresponding to three scenarios regarding the future structure of the European and global economies. The projections corresponding to the desired scenario are input into the macro-scale model which at each iteration allocates new activity to the COROPs and re-allocates existing activity on the basis of the relative competitiveness of the regions. The regional estimates are then converted via productivity functions into regional demands for cell space for the land 
uses corresponding to each activity (housing, industry, woodland, etc.); these demands constitute the constraints for the CA, which then determines the actual land use patterns. Information on regional densities and suitabilities is then returned from the CA to the macro-scale model, where it influences the cell demands generated in the next iteration.

The primary purpose of the model from the end user's point of view is to provide a tool for exploring the effects of alternative policy options on the quality of the environment. Since The Netherlands is a very densely populated country, environmental policies are as much concerned with the quality of the living environment of the people, e.g. access to green areas, nature reserves, and recreational areas, as it is with more traditional concerns such as preservation of species diversity. National policies concerning growth, development, and land use all have implications at the most local level, which is where many of the decisions that are most important in shaping the lived environment are taken. The integrated CA-based model thus functions to reveal the likely local consequences of the national policies, both in terms of land use as such, and also as shown by a number of economic, social, and ecological indicators that are calculated from the model output. Various policy options can be examined in this way, and the results used as the basis of discussions leading to policy formation.

\subsection{The CA land use model}

The CA component of the integrated model is defined on a grid of 351,000 $500 \times 500$ m cells. Sixteen land use and land cover categories are used in the cellular model - or occasionally more for certain applications. These are divided into three categories in terms of the way they are handled in the CA. Active land use categories are those for which the dynamics are fully modelled; their dynamics are driven by targets for cell totals supplied at each iteration by the regional model. Passive land use categories are those which are not driven by targets, so their dynamical behaviour is a residual of the dynamics of the active categories. Active and passive land uses together total some 132,000 cells. Finally, some land covers and land uses, like water and airports, are fixed. These feature cell states can only be changed by an intervention that is exogenous to the CA dynamics.

At each iteration, a vector of transition potentials is calculated for each cell in the array that is not in a state representing a fixed feature. These potentials represent the relative attractiveness of the cell for each of the non-feature land uses. More specifically, they reflect the attraction and repulsion effects of other land uses in the neighbourhood of the cell, as well as the effects of the accessibility of the cell to the road and rail networks (Fig. 1c), the suitability of the land for the particular use (Fig. 1a), and the land use zoning regulations (Fig. 1b). Transition potentials for each cell are calculated as follows:

$$
P_{j}=v A_{j} S_{j} Z_{j} N_{j}+H_{j}
$$

where $P_{j}=$ the potential of the cell for land use $j$; $v=$ a scalable random perturbation term; $A_{j}=$ accessibility of the cell to the road network; $S_{j}=$ the intrinsic suitability of 

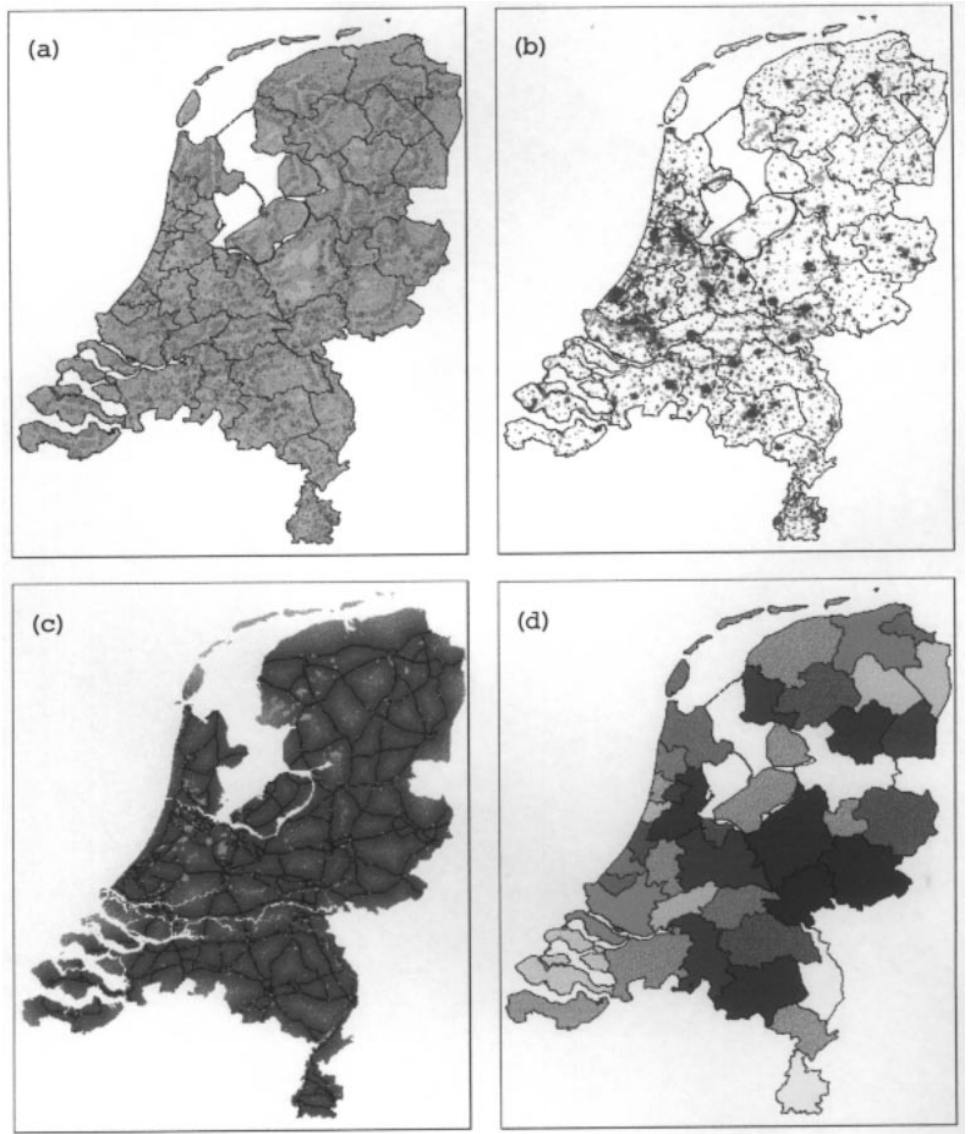

Fig. 1. Examples of input data for the LeefOmgevingsVerkenner model: (a) suitability for low density housing; (b) zoning map for low density housing; (c) accessibility values for low density housing; and (d) the 40 economic regions (COROPs).

the cell for land use $j ; Z_{j}=$ the zoning status of the cell for land use $j ; N_{j}=$ the neighbourhood effect on the cell for land use $j$; and $H_{j}=$ an inertia effect; $H_{j}>0$ if the current state is $j$; otherwise, $H_{j}=0$.

The neighbourhood effect is calculated over all cells within an eight-cell radius. Within this radius there are 30 discrete distance values, $d$. Cells within the neighbourhood are weighted according to their land use and their distance from the cell for which the potential is being calculated:

$$
N_{j}=\Sigma_{x} \Sigma_{d} w_{k x d} I_{x d},
$$

where $w_{k x d}=$ the weighting parameter applied to land use $k$ at position $x$ in distance zone $d$ of the neighbourhood; and $I_{x d}=$ the Dirac delta function: $I_{x d}=1$ if the cell is occupied by land use $k$; otherwise, $I_{x d}=0$. 
The network of major highways is represented by cell-centred vectors and appears superimposed on the cell grid. Accessibilities are calculated as a function of distance from the cell to the nearest point on the network:

$$
A_{j}=\left(1+D / a_{j}\right)^{-1}
$$

where $D=$ the Euclidean distance from the cell to the nearest cell through which the network passes; and $a_{j}=$ a coefficient representing the importance of accessibility to the network for land use $j$.

Once the vector of potentials has been calculated for all non-feature cells, state transitions are determined. First, the number of cells in each state for each region is determined from the regional model. Then, in each region in which the cell demands can be accommodated, each cell is assigned the state for which it has the highest potential as determined by Eq. (1), with cells being assigned states in the order of the ranked potentials, starting with the highest. However, once the target number of cells for a given land use has been attained, potentials for that state are ignored when determining the state of subsequent cells. If all cell demands within a region cannot be satisfied, the productivity function relating activity level to cell demand (e.g. population to housing cells required) increases the densities in that region, thus reducing cell demand. If that is still not sufficient, then the excess cells that cannot be accommodated in the region are added to the cell demands of other regions where adequate space is available.

\subsection{The linked regional model}

The model which generates activity levels for the various economic sectors as well as population in each of the 40 COROPs (Fig. 1d) is based on classical spatial interaction equations, supplemented by terms which capture the effect of densities, average suitability levels, and average cellular potential levels within the regions. We show here one equation to illustrate the nature of the model and display the features relevant to integration with the CA.

First, the model allocates to the COROPs new activity due to national growth, and reallocates some portion of the existing activity among the regions in order to represent the effect of competition among the regions. For example, migration of activity $K$ into region $i$ from all other regions $j$ is given by:

$$
\begin{array}{r}
{ }^{t} D_{I k i}=\sum_{j \neq i}\left[{ }^{t-1} X_{K j}^{\prime}\left(\frac{{ }^{t} X_{p i} / d_{i j}^{n}}{{ }^{t} V_{P j}}\right)^{\beta K 1}\left(\frac{{ }^{t} J_{i} / d_{i j}^{n}}{{ }^{t} V_{J j}}\right)^{\beta K 2}\left(\frac{{ }^{t} X_{K i} / d_{i j}^{n}}{{ }^{t} V_{K j}}\right)^{\beta K 3}\left(\frac{{ }^{t} W_{K j}^{\prime}}{{ }^{t} W_{K i}^{\prime}}\right)^{\beta K 4}\right. \\
\left.\left(\frac{\left\langle\operatorname{pot}_{K i}\right\rangle}{\left\langle\operatorname{pot}_{K j}\right\rangle}\right)^{\beta K 6}\left(\frac{\left\langle\operatorname{suit}_{K i}\right\rangle}{\left\langle\operatorname{suit}_{K j}\right\rangle}\right)^{\beta K 7}\right],
\end{array}
$$

where ${ }^{t} D_{I K i}=$ demand for migration of activity $K$ into region $i$ at time $t ; X_{K j}=$ level of activity $K$ in region $j$ and $X_{P j}=$ population in region $j ; J_{i}=$ jobs in region $j$; 
$d_{i j}=$ distance between regions $i$ and $j ; V_{K j}=$ the potential at region $j$, a measure of the accessibility of region $j$ to activity $K$ in all regions, calculated as:

$$
{ }^{t} V_{K i}=\sum_{j=1}^{R}\left(\frac{{ }^{t} X_{K j}}{d_{i j}^{n}}\right)
$$

and similarly for $V_{p}$ and $V_{J} ; W_{K j}=$ the density of activity $\mathrm{K}$ per cell in region $j ;\left\langle\operatorname{pot}_{K j}\right\rangle=$ the mean cellular potential for the land use corresponding to activity $K$ in region $j$; $\left\langle\right.$ suit $\left._{K j}\right\rangle=$ the mean suitability for the land use corresponding to activity $K$ in region $j$; and $\beta_{K i}=$ parameters to be calibrated.

A similar equation represents flows out of the region, and so the net desired change in activity $K$ in the region is given by the difference of the two quantities, plus the regional allocation of new activity. It is worth emphasizing that these are only demands for the location of activity, since the actual relocation of activity can only take place to the extent that space is available in the region, and that is determined in the cellular model.

The most significant feature of Eq. (4) in terms of integrated modelling is the inclusion of the three terms representing cellular-level features. The densities are cellular densities, and thus a good measure of actual crowding in the region. If density were to be measured as simply the amount of activity in a region divided by the area of the region, the result in many cases would be seriously to misrepresent the actual situation. By such a measure, for example, a dense urban region with a small amount of industry would show a low density of industry; but the industry actually located there would be experiencing the disadvantages of congestion and high land prices associated with high densities. Similarly, the cellular potential term captures the desirability of the land actually occupied by the activity in region $j$ relative to that in region $i$; this measure summarizes the neighbourhood effect as well as accessibility, suitability, and zoning, and is a good indication of the quality of location that new activity moving into the region could expect. The suitability term is primarily applicable to the agricultural sectors; for other activities, $\beta_{K 7}$ is set to zero. In sum, then, including the cellular-level terms in the regional model allows the macro-scale model to take into account the particular, very local conditions that are actually experienced by the individual actors who make locational decisions, and that in aggregate constitute the various activity sectors.

Having established demands for activity levels in each region, these must be translated into demands for land that can be used as constraints in the CA. At each iteration, the productivities or densities, $W_{K j}$, are adjusted in function of the demand for each activity relative to the current level of activity, as well as the trends in potentials and suitabilities. Thus, higher demands for activity tend to increase land productivities, and declining mean potentials and suitabilities tend to decrease them. The new productivities are then used to establish the cell demands, which are passed to the CA. If the CA cannot satisfy the cell demand in a region, it reallocates portions of the cell demands for some land uses to other regions. Land uses with lower potentials have a higher priority for reallocation to other regions. Destination 
regions for each activity for which reallocation is necessary are chosen on the basis of demand relative to current level of activity, with cell demands reallocated first to those regions with the highest relative demand for the activity. Finally, the actual new activity levels in the regional model are calculated as the products of the new regional cell totals and the new productivities. Thus, the CA is in a sense incorporated into the regional model, and vice versa, so that the integration is complete.

The full model has been calibrated to 1989 data, and run to 1993. Since both land use data and COROP populations are available for the latter year, it is possible to test the performance of the model with respect to these aspects. In one set of experiments, the regional model was run decoupled from the CA to produce COROP population estimates. Comparing these runs with the results of the fully integrated model, it is found that linking the CA component improves COROP population estimates by between 65 and $70 \%$. Attempts to assess the quality of the land use predictions have been delayed due to problems with the 1993 data set, which are now being corrected. The initial land use map (1989) and the land use for the final year of the full simulation (2029) are shown in Fig. 2.

\section{General issues raised by high-resolution integrated modelling}

The very power and realism of CA-based models raise other, very general issues which are only beginning to be recognized. One immediate problem is how to assess the output of these models. A good CA-based model produces results which have all the patterned complexity of the real system. In order to test such a model, it is

(a)

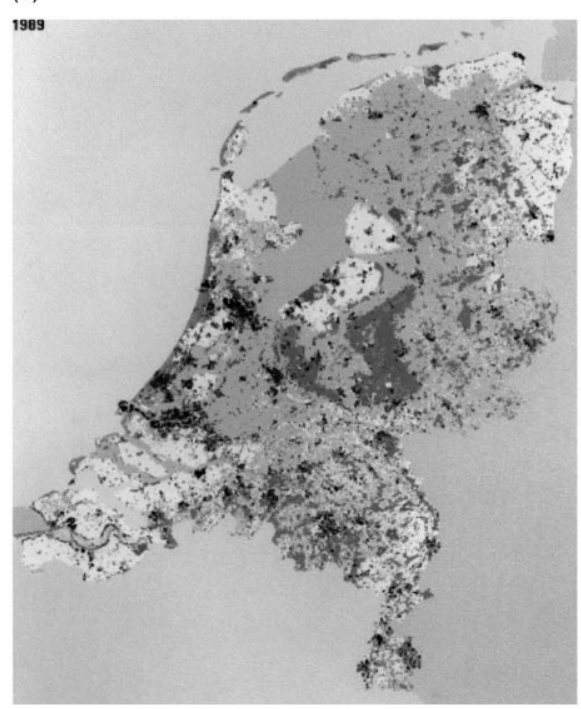

(b)

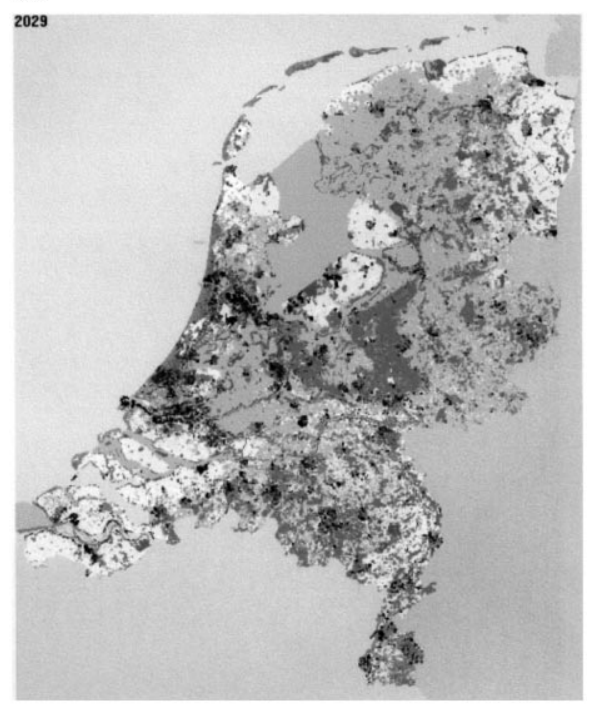

Fig. 2. Initial and final land uses: (a) actual land use, 1989; and (b) simulated land use, 2029. 
necessary to compare the simulation results with the actual data. But immediately we are faced with the problem of how to compare the two maps. One approach is to use very general measures, such as the various fractal dimensions that have been found to characterize certain geographical structures, most notably cities (Frankhauser, 1994; White \& Engelen, 1993). While these sorts of measures are extremely useful for certain purposes, they are not in themselves sufficient. First, they have not been shown to be applicable to non-urban situations. More importantly, they are extremely abstract measures: e.g. two maps which appear completely different may have identical fractal dimensions; thus they tell us very little about how similar the two maps may be in terms of their local configurations.

At the other extreme are pixel-by-pixel map comparisons and the general measures based on them, like the Kappa index. A pixel-by-pixel approach is appropriate for most standard data quality problems: e.g. every pixel of a classified satellite image should match the ground truth. But it is mistaken to expect the output of a CA model to match the actual data cell by cell, even in principle. Most realistic CA include a stochastic element in order to capture the inherent stochasticity of both natural and human systems. Thus, not only is any particular simulation result only one of a large number that are possible with the same initial conditions and a given parameterization, but also the actual data set with which the result is being compared represents only one of an infinite number of possible configurations, although most of these are presumably highly similar. Thus, many disagreements between the two maps represent simply the stochasticity in the two systems being compared.

Furthermore, since spatial configurations have functional significance in geographical systems, a more relevant question is whether the patterns of the two maps are similar. One way to approach this issue is to use a polygon-by-polygon comparison, in which various characteristics of the polygons can be compared, starting with the extent to which polygons on one map coincide with those on the other, but also including other measures of polygon similarity such as size and compactness. A polygon-based technique can produce as a first output a map showing levels of agreement between the maps being compared, and thus indicate areas or features which cause difficulties for the model; it is also possible to generate a global similarity measure. A polygon-based approach using fuzzy set techniques has been implemented by Power, Simms and White (2000) with good results, but the map comparison problem remains a large and open issue. The field of pattern recognition is likely to be a source of further developments in this area.

A related but much more fundamental issue concerns the nature of predictability in geographical systems. It is well understood that these systems are highly complex in their behaviour, characterized not only by stochasticity, but also deterministic non-linear dynamics which can mimic stochasticity. Furthermore, like all selforganizing systems, they undergo continual structural evolution, an evolution typically characterized by periods of slow, continuous change punctuated by episodes of dramatic change when the system passes through a bifurcation point. At a bifurcation, the probability distribution of system states becomes strongly bi-modal, and as the system moves beyond the bifurcation it will become locked into one or another of the two bundles of possible trajectories. In other words, each time the system 
passes through a bifurcation point it must choose one or another of two quite different futures. Thus, geographical systems, despite being governed by largely deterministic processes, have open, undetermined futures. The phenomenon is increasingly well understood at a theoretical level, and some modelling techniques address it directly. But the methodological standards and procedures for hypothesis testing and theory and model validation are still based on the older, much simpler view of determinism, and lag far behind.

Current models are far from capturing all of these levels of behaviour. CA-based integrated models represent dynamics and stochasticity explicitly, and with proper sensitivity analysis can give some insight into the possibility and consequences of future structural evolutionary changes in the system, although these are not modelled directly. But since the systems being modelled are dynamic, evolving, creative, with a future that is open, an appropriate model should also have these characteristics. Then the question is to what extent the bundle of possible outcomes of the model coincides with the possible futures of the real system. The traditional hypothesis-testing methodology is not entirely useful in this situation, and more appropriate methodologies are yet to be worked out. This is a problem that is attracting increasing attention in a number of fields, most notably physics and biology.

\section{Conclusion}

CA were originally developed to investigate the behaviour of dynamical systems, and many geographical applications still emphasize primarily theoretical issues. Such work is necessary, because there remains much to learn about the dynamics of spatial systems. Increasingly, however, CA are being developed which demonstrate a capacity for modelling real geographical systems with precision and realism. These applications suggest that in the next decade CA-based models may begin to find a place as practical policy and planning tools. Nevertheless, the world is a complex mix of predictability, uncertainty, and novelty. The integrated CA modelling approach begins to capture this, but at the same time it implies a different kind of science for which the methodology and standards have not yet been fully worked out.

\section{Acknowledgements}

The models described in this paper were developed in part with the assistance of funding from the Rijksinstituut voor Volksgezondheid en Milieu (RIVM) and the Social Sciences and Humanities Research Council of Canada (SSHRC).

\section{References}

Batty, M., \& Xie, Y. (1994). From cells to cities. Environment and Planning B, 21, s31-s48.

Batty, M., \& Xie, Y. (1996). Possible cellular automata. In E. Besussi, \& A. Cechini, Artificial worlds and urban studies (pp. 191-220). Venice: DAEST. 
Benenson, I. (1998). Multi-agent simulations of residential dynamics in the city. Computers, Environment and Urban Systems, 22, 25-42.

Bura, S., Guerin-Pace, F., Mathian, H., Pumain, D., \& Sanders, L. (1996). Multiagent systems and the dynamics of a settlement system. Geographical Analysis, 28, 161-175.

Cecchini, A. (1996). Approaching generalized automata with help on line (AUGH). In E. Besussi, \& A Cechini, Artificial worlds and urban studies (pp. 231-248). Venice: DAEST.

Cecchini, A., \& Viola, F. (1990). Eine Stadtbausimulation. Wissenschaftliche Zeitschrift der Hochschule fur Architektur und Bauwesen, 36, 159-162.

Clarke, K., Hoppen, S., \& Gaydos, I. (1997). A self-modifying cellular automaton model of historical urbanization in the San Francisco Bay area. Environment and Planning B, 24, 247-261.

Couclelis, H. (1985). Cellular worlds: a framework for modelling micro-macro dynamics. Environment and Planning A, 17, 585-596.

Couclelis, H. (1988). Of mice and men: what rodent populations can teach us about complex spatial dynamics. Environment and Planning A, 20, 99-109.

Couclelis, H. (1989). Macrostructure and microbehavior in a metropolitan area. Environment and Planning $B, 16,151-154$.

Couclelis, H. (1997). From cellular automata to urban models: new principles for model development and implementation. Environment and Planning B, 24, 165-174.

Dong, X. (2000). A cell based simulation model of the urban land market. Unpublished thesis, Memorial University of Newfoundland.

Engelen, G., Uljee, I., \& White, R. (1997). Vulnerability assessment of low-lying coastal areas and small islands to climate change and sea level rise. Report to United Nations Environment Program, CAR/ RCU. Maastricht: Research Institute for Knowledge Systems.

Engelen, G., White, R., \& Uljee, I. (1993). Exploratory modelling of socio-economic impacts of climatic change. In G. A. Maul, Climatic change in the intra-Americas Sea (pp. 350-368). London: Edward Arnold.

Engelen, G., White, R., \& Uljee, I. (1997). Integrating constrained cellular automata models, GIS and decision support tools for urban planning and policy-making. In H. Timmermans, Decision support systems in urban planning (pp. 125-155). London: E\&FN Spon.

Engelen, G., White, R., \& Uljee, I. (1998). De LeefOmgevingsVerkenner. 'Proof of Concept'-versie van een intgraal model voor het berekenen van het LeefOmgevingsKapitaal in Nederland. Maastricht: Research Institute for Knowledge Systems.

Engelen, G., White, R., Uljee, I., \& Wargnies, S. (1996). Numerical modeling of small island socioeconomics to achieve sustainable development. In G. A. Maul, Small islands. marine science and sustainable development (pp. 437-463). Coastal and Estuarine Studies, 51. Washington DC: American Geophysical Union.

Frankhauser, P. (1994). La fractilite des structures urbaines. Paris: Economica.

Itami, R. (1994). Simulating spatial dynamics: cellular automata theory. Landscape and Urban Planning, 30, 27-47.

Kauffman, S. (1989). Adaptation on rugged fitness landscapes. In P. Stein, Lectures in the sciences of complexity (pp. 527-618). Reading, MA: Addison-Wesley.

Nagel, K., Rasmussen, S., \& Barrett, C. (1997). Network traffic as a self-organized critical phenomenon. In F. Schweitzer, \& H. Haken, Self-organization of complex structures: from individual to collective dynamics (pp. 579-592). Amsterdam: Gordon and Breach.

Papini, L., \& Rabino, G. (1997). Urban cellular automata: an evolutionary prototype. In S. Bandini, \& G. Mauri, ACRI'96: Proceedings of the second conference on cellular automata for research and industry (pp. 147-157). Berlin: Springer.

Phipps, M. (1989). Dynamical behaviour of cellular automata under constraints of neighbourhood coherence. Geographical Analysis, 21, 197-215.

Phipps, M. (1992). From local to global: the lesson of cellular automata. In D. DeAngelis, \& L. Gross, Individual based models and approaches in ecology: populations, communities and ecosystems (pp. 165187). New York: Routledge, Chapman and Hall. 
Phipps, M., \& Langlois, A. (1997). Spatial dynamics, cellular automata, and parallel processing computers. Environment and Planning B, 24, 193-204.

Portugali, J., \& Benenson, I. (1995). Artificial planning experience by means of a heuristic cell-space model: simulating international migration in the urban process. Environment and Planning A, 27, 16471665 .

Portugali, J., \& Benenson, I. (1997). Human agents between local and global forces in a self-organizing city. In F. Schweitzer, \& H. Haken, Self-organization of complex structures: from individual to collective dynamics (pp. 537-545). Gordon and Breach.

Portugali, J., Benenson, I., \& Omer, I. (1994). Socio-spatial residential dynamics: stability and instability within a self-organizing city. Geographical Analysis, 26, 321-340.

Portugali, J., Benenson, I., \& Omer, I. (1997). Spatial cognitive dissonance and sociospatial emergence in a self-organizing city. Environment and Planning B, 24, 263-285.

Power, C., Simms, A., \& White, R. (2000). Hierarchical fuzzy pattern matching for the regional comparison of land use maps. International Journal of Geographical Information Systems (in press).

RIVM (1998). The environment explorer: a prototype on trial. Bilthoven: Rijksinstituut voor Volksgezondheid en Milieu.

Sanders, L., Pumain, D., \& Mathian, H. (1997). SIMPOP: a multiagent system for the study of urbanism. Environment and Planning B, 24, 287-305.

Theobald, D., \& Gross, M. (1994). EML: a modeling environment for exploring landscape dynamics. Computers, Environment, and Urban Systems, 18, 193-204.

Tobler, W. (1979). Cellular geography. In S. Gale, \& G. Olsson, Philosophy in Geography (pp. 379-386). Dordrecht: Reidel.

Uljee, I., Engelen, G., \& White, R. (1996). RamCo Demo Guide (Workdocument CZM-C 96.08). The Hague: Coastal Zone Management Centre, National Institute for Coastal and Marine Management.

Wagner, D. (1997). Cellular automata and geographic information systems. Environment and Planning B, 24, 219-234.

White, R., \& Engelen, G. (1993). Cellular automata and fractal urban form: a cellular modelling approach to the evolution of urban land use patterns. Environment and Planning A, 25, 1175-1199.

White, R., \& Engelen, G. (1994). Cellular dynamics and GIS: modelling spatial complexity. Geographical Systems, 1, 237-253.

White, R., \& Engelen, G. (1997a). Cellular automata as the basis of integrated dynamic regional modelling. Environment and Planning B, 24, 235-246.

White, R., \& Engelen, G. (1997b). Multi-scale spatial modelling of self-organizing urban systems. In F. Schweitzer, \& H. Haken, Self-organization of complex structures: from individual to collective dynamics (pp. 519-535). Amsterdam: Gordon and Breach.

White, R., Engelen, G., \& Uljee, I. (1997). The use of constrained cellular automata for high-resolution modelling of urban land use dynamics. Environment and Planning B, 24, 323-343.

White R., Engelen, G. \& Uljee, I. (2000). Modeling land use change with linked cellular automata and socio-economic models: a tool for exploring the impact of climate change on the island of St. Lucia. In M. Hill, \& R. Aspinall, Spatial information for land use management. Gordon and Breach (in press).

$\mathrm{Wu}, \mathrm{F}$. (1998a). An experiment on the generic polycentricity of urban growth in a cellular automatic city. Environment and Planning B, 25, 731-752.

Wu, F. (1998b). SimLand: a prototype to simulate land conversion through the integrated GIS and CA with AHP-derived transition rule. International Journal of Geographical Information Science, 12, 63-82.

Xie, Y. (1996). A generalized model for cellular urban dynamics. Geographical Analysis, 28, 350-373. 